\title{
A qualitative exploration into the factors that influence Irish teens' food choices
}

\author{
A.N. Daly ${ }^{1}$, J.M. Kearney ${ }^{1}$ and E.J. O'Sullivan ${ }^{1}$ \\ ${ }^{1}$ School of Biological \& Health Sciences, City Campus, Technological University Dublin, Dublin, Ireland
}

The aim of this research was to investigate the attitudes, behaviours and influences surrounding food choices and eating behaviours among Irish teens. Qualitative methods were used to gain a deeper understanding from the teens' perspective, based on their lived experiences around food.

Six semi-structured focus group discussions were completed with Irish secondary school-going teens in Transition Year, aged 15-17 years $(n=47)$. Participants came from both single-sex and mixed schools in Dublin and Wexford, and included both males and females. Due to Covid-19 restrictions, five focus groups were conducted via video call. Discussion topics were introduced using a vignette format, with the content based on key eating behaviour styles identified in the literature: restrained, emotional, uncontrolled eating $^{(1)} \&$ variety seeking tendencies ${ }^{(2)}$. Focus groups were moderated by the first author and attended by a research assistant, who took detailed notes. Data coding was managed using NVivo software (V12) and analysis was completed following the six phases of reflexive Thematic Analysis, as outlined by Braun and Clarke ${ }^{(3)}$.

Three themes relating to teen food choices and their eating behaviours were generated: 1) the importance of enjoying food and the eating occasion, 2) the role of the food environment, and 3) social concerns around health and appearance. Of overwhelming importance was the enjoyment factor from food, in terms of both taste and the overall experience of the eating occasion. The food environment includes food availability at school, at home and the social environment and concerns around food and eating with peers. Concerns for physical and social appearance, as well as price, convenience and familiarity with the food were factors involved in food choices, but the priority for all teens was to enjoy the food and the occasion around eating. Health was mentioned as a factor, but more in relation to the perception of health and what was socially acceptable, where eating certain foods could be perceived as being "healthy/good" or "unhealthy/bad". Teens described concerns about social judgement associated with certain foods, rather than concerns about their impact on the teen's physical health. In general, the participants in this research had a strong understanding of nutrition and the social pressures linked with food and body image, but did not feel that they themselves fall victim to peer judgement or pressure from social media directly.

The thoughts involved when making food choices encompass a wide range of factors for teens, and decisions regarding what to eat are linked to the individual priorities of the teen themselves and are highly context specific and fluid. Taste and enjoyment are highly important, but concerns for the social acceptability of certain foods can play an important and often conflicting role.

\section{References}

1. Karlsson J, Persson LO, Sjostrom L, et al. (2000) Int J Obes Relat Metab Disord. 24(12), 1715-25.

2. Van Trijp HCM \& Steenkamp J-BEM (1992) Eur Rev Agric Econ 19(2), 181-95.

3. Braun V \& Clarke C (2020) Qual Res Psychol. 